\title{
Wilms Tumour with an Unusual Urothelial Differentiation and Teratoid Components
}

\author{
Aravind Sekar ${ }^{1 *}$, Anand Bardia ${ }^{2}$, Srinivasan Peyam ${ }^{3}$, Amita Trehan ${ }^{3}$ \\ ${ }^{1}$ Assistant Professor, Department of Histopathology, PGIMER, Chandigarh, India \\ ${ }^{2}$ Department of Histopathology, PGIMER, Chandigarh, India \\ ${ }^{3}$ Department of Paediatric Haematology and Oncology, PGIMER, Chandigarh, India
}

\author{
DOI: $10.36348 /$ sjpm.2021.v06i02.003 $\quad$ | Received: 01.01.2021 | Accepted: 04.02.2021 | Published: 11.02 .2021 \\ *Corresponding author: Dr. Aravind Sekar
}

\section{Abstract}

Wilms tumours with a greater number of heterologous elements on histology are considered as teratoid form of Wilms tumour. The presence of teratoid components in Wilms tumour are resistant to chemotherapy and may warrant additional treatment. On microscopy, the epithelial component in Wilms tumour most commonly present in form of tubules or glomeruloid bodies as homologous differentiation and less commonly as heterologous elements such as squamous or mucinous epithelium. Other common heterologous elements described in the literature are skeletal muscle, smooth muscle, adipose tissue and cartilage. Though the transitional lining is homologous to the kidney, nests of urothelial cells within Wilms tumour is not described well in the literature. We describe the presence of urothelial nests intimately admixed with other homologous and heterologous components of Wilms tumour in post-chemotherapy nephrectomy specimen.

Keywords: Teratoid, Wilms tumour, Urothelial nests.

Copyright () 2021 The Author(s): This is an open-access article distributed under the terms of the Creative Commons Attribution 4.0 International License (CC BY-NC 4.0) which permits unrestricted use, distribution, and reproduction in any medium for non-commercial use provided the original author and source are credited.

\section{INTRODUCTION}

Wilms tumour is the most common paediatric renal tumours and constitutes $6 \%$ of all childhood tumours [1]. On histology, it shows different proportions of blastemal, epithelial and stromal components with variable degrees of heterologous elements. Most common heterologous elements described in the literature were skeletal muscle, smooth muscle, adipose tissue, cartilage, squamous epithelium. Wilms tumours with a greater number of heterologous elements on histology were considered as a teratoid form of Wilms tumour, nonetheless, there are no definite criteria. In addition, although the transitional lining is homologous to the kidney, nests of urothelial cells within the Wilms tumour are not well documented in the literature. We describe a case of Wilms tumour, which had shown heterologous components and the presence of an unusual urothelial component in postchemotherapy nephrectomy specimen.

\section{CASE STUDY}

A 3-year-old boy, firstborn to a nonconsanguineous couple, presented with a palpable lump over the left upper abdomen by his mother while bathing him and dull aching pain for 20 days. On physical examination, his blood pressure was 128/79 $\mathrm{mmHg}$ (> 95th percentile) and a well-defined, firm, non-tender, bi-manually palpable mass of $7 * 8 \mathrm{~cm}$ in the left lumbar region, with no other obvious findings or clinical stigmata of WT-1 related syndromes. CECT abdomen revealed a large well-defined encapsulated exophytic isodense mass of $8.7 * 7.0 * 6.5 \mathrm{~cm}$ in the milower pole of the left kidney with no renal vessel thrombosis. CT chest revealed no lung nodules. Fine needle aspiration cytology from the mass was suggestive of a Wilms tumour with triphasic components. He was initiated on pre-operative twodrug chemotherapy in accordance with SIOP-WT$2 \mathrm{OO} 1$ localised disease protocol. He received 4 weeks of chemotherapy (weekly dose vincristine @ 1.5 $\mathrm{mg} / \mathrm{m} 2 \times 4$ doses \& 2-weekly doses of actinomycin-D @ $45 \mu \mathrm{g} / \mathrm{kg} \times 2$ doses). A reassessment of the CECT abdomen showed a mass of $8.5 * 6 * 5 \mathrm{~cm}$ with a large cystic/necrotic component with a small area of the solid component. He underwent an open left radical nephroureterectomy. The tumour was adherent to the left psoas muscle but grossly excised. Grossly, 
nephrectomy specimen showed an encapsulated tumour with solid $(60 \%)$ and cystic $(60 \%)$ components, measuring $6 \mathrm{~cm}$ in maximum dimension (Fig-1). Renal pelvis and sinus were free of tumour.

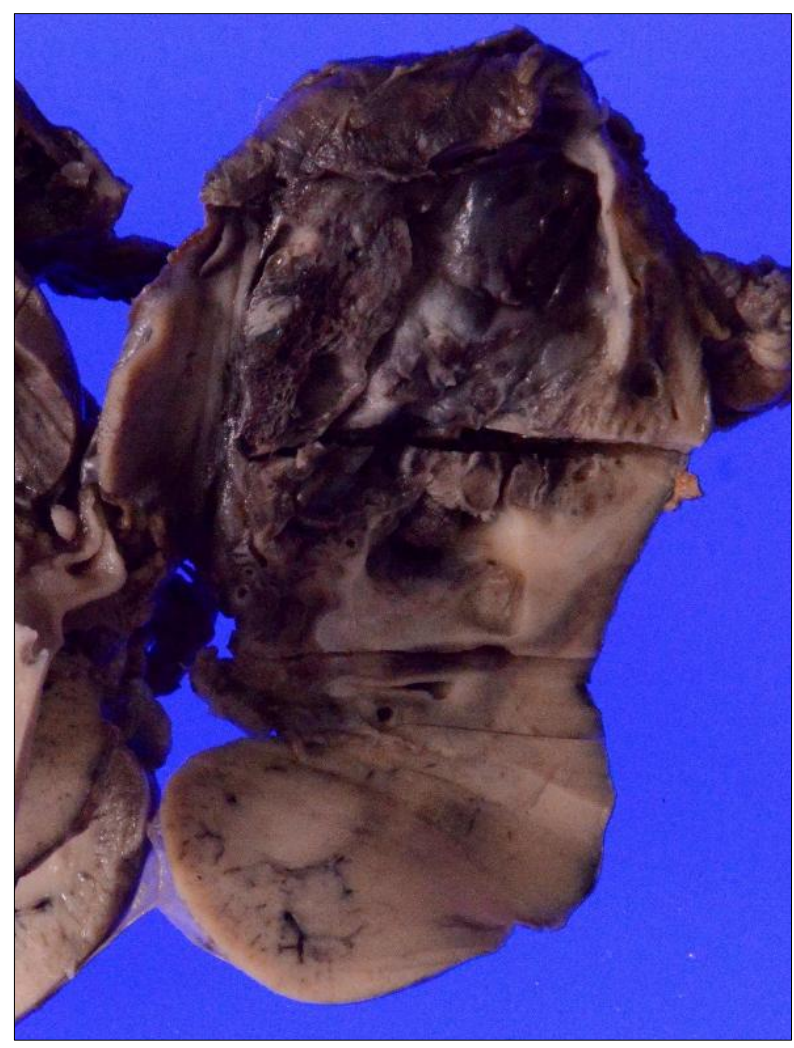

Fig-1: Cut surface of left kidney showing solid cystic tumour with areas of Haemorrhage and necrosis (Gross photograph)

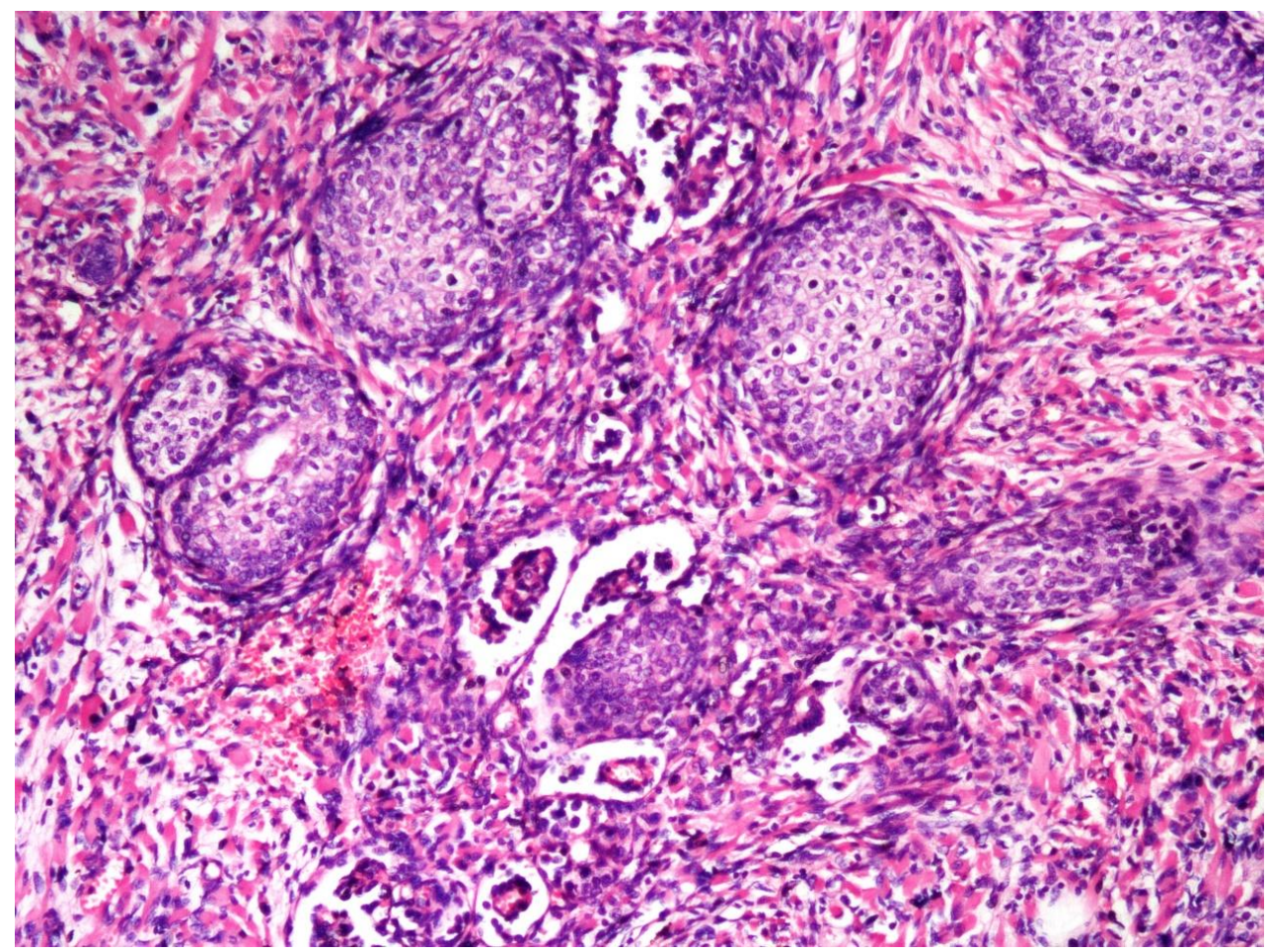

Fig-2: Nests of urothelial cells admixed with glomeruloid bodies and small tubules (H\&E, original magnification x200) 


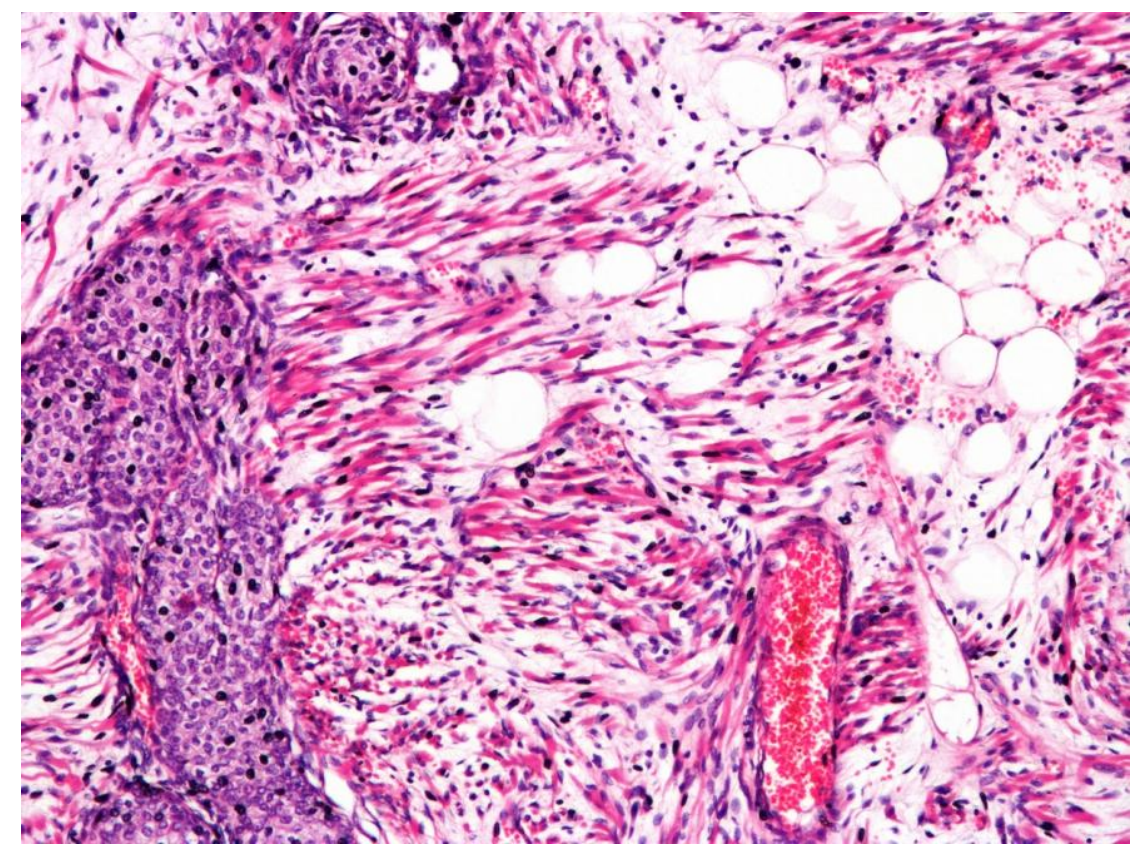

Fig-3: Heterologous elements comprising lobules of adipose tissue, fascicles of smooth muscle fibres along with urothelial component (H\&E, original magnification $\times 200$ )

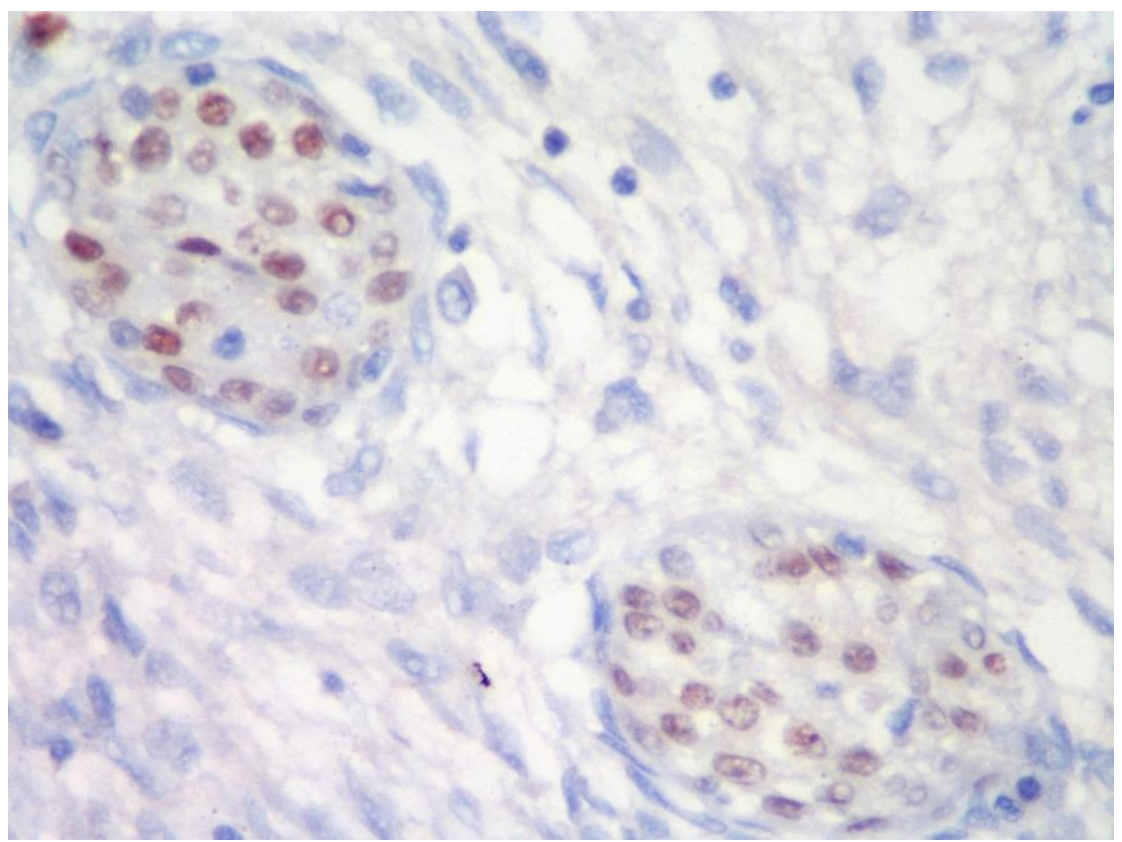

Fig-4: Nuclear positivity for GATA 3 is noted in urothelial nests (Immunohistochemistry, x200)

Multiple sections examined from the solid areas show a triphasic tumour comprising predominantly epithelial $(60 \%)$, blastemal $(<5 \%)$ and mesenchymal components $(35 \%-40 \%)$. The epithelial component was seen in the form of glomeruloid bodies and tubules. Heterologous components such as adipocytes, fascicles of smooth muscle fibres were seen in $60 \%$ of the mesenchymal component. Besides, we observed nests of urothelial cells (Fig-2) in the tumorous areas (10\% of the epithelial component), intimately admixed with glomeruloid bodies, tubules, lobules of adipocytes and fascicles of smooth muscle fibres (Fig-3). On Immunohistochemistry, urothelial nests had shown nuclear positivity for GATA3 (Fig- 4).
There are no anaplastic features or nephrogenic rest or lymphovascular invasion or capsular breach observed in the sections. Regional lymph nodes are free of tumour. According to ISOP post-chemotherapy protocol (3), the tumour is stratified in the Wilms tumour, mixed type (intermediate risk) group, stage II. Observation of predominant lobules of adipocytes and smooth muscles in the index case may qualify for the teratoid Wilms tumour entity and in those findings of urothelial nests are unusual. He was initiated on post-operative AV-2 two-drug chemotherapy (Vincristine plus ActinomycinD), which is of 27 weeks of duration. Currently, the child is clinically well and has completed 20 weeks of adjuvant chemotherapy. 


\section{DISCUSSION}

The term teratoid Wilms tumour implies resemblance to the teratoid tumour with multiple lineages of differentiation not indigenous to the site. It differs from very rare cases of intrarenal teratoma by the presence of other classical Wilms tumour components such as blastemal elements and glomeruloid bodies. Teratoid form of Wilms tumour was first observed and reported by Variender et al., in 1984 [2]. Following which, many cases of teratoid Wilms tumour with diverse epithelial and mesenchymal components were documented in the literature. Frequent teratoid elements observed in the Wilms tumour are skeletal muscle, smooth muscle, adipose tissue, cartilage, bone [3].

The pathogenesis of formation of teratoid elements in the Wilms tumour is still unclear and probably it may originate from totipotent primitive metanephric mesenchyme [4]. The intracellular matrix proteins present in the tumour may influence the occurrence, degree, and diversity of heterologous differentiation [5]. It is also proven from the observation that heterologous elements are more commonly observed in stromal rich Wilms tumour [6].

Though SIOP and COG Wilms tumour protocols excluded teratoid features in their treatment stratification criteria as the prognosis depends only on the histological type and stage [7], many authors emphasise that presence of teratoid components is chemotherapy-resistant and may warrant additional treatment. Hooge et al., considered that these teratoid elements were extreme end of heterologous element differentiation and showed significant associations with younger age, bilateral disease, intralobar nephrogenic rests, and stromal predominant Wilms tumour [8]. In the index case, the presentation is at a younger age with unilateral involvement and there was mild shrinkage of tumour size with areas of necrosis and cystic degeneration after chemotherapy in reassessment CECT of abdomen. Mixed type histology with admixture of stromal, epithelial and blastemal elements were observed in the post chemotherapy nephrectomy specimen and categorised as the intermediate risk group.

The diversity in epithelial differentiation is rare. The most common one reported in the literature were Squamous epithelium with keratin pearls followed by mucinous epithelium $[9,10]$. Though the transitional lining is homologous to the kidney and originates from the ureteric bud during embryogenesis, nests of urothelial cells within Wilms tumour is not well described in the literature. Urothelial nests observed in this case are intimately admixed with other heterologous elements such as adipose tissue and fascicles of smooth muscle fibres and far from pelvic calyceal system. The presence of urothelial nests in
Wilms tumour is new fangled for us and may be originate from totipotent stem cells in the primitive mesenchyme. The significance of finding of urothelial nests along with other heterologous elements in the post chemotherapy nephrectomy specimen might indicate its resistance to chemotherapy. It should be documented in the report separately as it may necessitate additional treatment.

Conflict of Interest: No conflict of interest.

\section{Funding: None}

\section{REFERENCES}

1. Guruprasad, B., Rohan, B., Kavitha, S., Madhumathi, D. S., Lokanath, D., \& Appaji, L. (2013). Wilms' tumor: single centre retrospective study from South India. Indian journal of surgical oncology, 4(3), 301-304.

2. Variend, S., Spicer, R. D., Lond, L., \& Mackinnon, A. E. (1984). Teratoid Wilms' tumor. Cancer, 53(9), 1936-1942.

3. Inoue, M., Uchida, K., Kohei, O., Nashida, Y., Deguchi, T., Komada, Y., \& Kusunoki, M. (2006). Teratoid Wilms' tumor: a case report with literature review. Journal of pediatric surgery, 41(10), 1759-1763.

4. Beckwith, J. B., Kiviat, N. B., \& Bonadio, J. F. (1990). Nephrogenic rests, nephroblastomatosis, and the pathogenesis of Wilms' tumor. Pediatric pathology, 10(1-2), 1-36.

5. Sariola, H., Ekblom, P., Rapola, J., Vaheri, A., \& Timpl, R. (1985). Extracellular matrix and epithelial differentiation of Wilms' tumor. The American journal of pathology, 118(1), 96-107.

6. Gupta, R., Sharma, A., Arora, R., \& Dinda, A. K. (2009). Stroma-predominant Wilms tumor with teratoid features: report of a rare case and review of the literature. Pediatric surgery international, 25(3), 293-295.

7. Vujanić, G. M., Gessler, M., Ooms, A. H., Collini, P., Coulomb-l'Hermine, A., D'Hooghe, E., ... \& Graf, N. (2018). The UMBRELLA SIOP-RTSG 2016 Wilms tumour pathology and molecular biology protocol. Nature reviews Urology, 15(11), 693-701.

8. D’Hooghe, E., Mifsud, W., \& Vujanić, G. M. (2019). "Teratoid" Wilms Tumor: The Extreme End of Heterologous Element Differentiation, Not a Separate Entity. The American journal of surgical pathology, 43(11), 1583-1590.

9. Yadav, Y. K., Sharma, U., Gupta, K., \& Arora, R. (2012). Squamous predominant teratoid Wilms' tumor. Journal of laboratory physicians, 4(1), 5052.

10. Parikh, B., Trivedi, P., \& Shukla, K. (2007). A unilateral teratoid Wilms' tumor with raised serum alpha-fetoprotein level. Indian journal of pathology \& microbiology, 50(2), 317-319. 